\title{
Reflections on our first year
}

\author{
It has been a year since Nature Aging published its first issue. We now reflect on this past year by highlighting \\ some of the exciting content we have published. We also take this opportunity to share some of our experience in \\ launching a journal during a pandemic and to extend our gratitude to the community.
}

A year ago, Nature Aging published its inaugural issue. Our first Editorial stated that the journal would aim to feature research from across the entire spectrum of aging research. One year later, it is rewarding to look back at our content and see that it indeed reflects such diversity. Across our twelve issues in 2021, we published 66 primary research articles and 81 reviews and opinion articles. These papers covered the biology of aging and age-related diseases in both animals and humans, and included clinical, public health and social science research. The cover art for each issue and associated cover lines offer a visual snapshot of such research diversity. To celebrate our anniversary, we reflect on our work with the community over the past year by highlighting some of the articles we published.

Many papers in our first volume addressed questions related to the biology of aging and longevity. A pair of papers in our first issue looking at the role of dietary protein intake on healthspan and lifespan in flies or in mice were highlighted in a News \& Views article in Nature. In a December article, scientists showed that vaccination against a new seno-antigen could delay aging and increase lifespan in mice. An exome-sequencing paper in our September issue reported several rare coding variants associated with extreme human longevity. In our March issue, a longitudinal human cohort study revealed disparities in the pace of biological aging in midlife. We also published work that developed new tools and resources for aging research, including a new statistical framework to estimate epigenetic age at the single-cell level and a new mouse model to study senescence.

Among the diseases of aging, dementia, including Alzheimer's disease (AD), is one of the most common conditions in older adults and causes an enormous burden on our society. A few outstanding dementia studies were published in the journal. One of our first publications measured the prognostic value of blood-based biomarkers for cognitive decline in patients with $\mathrm{AD}$, which is currently our most-accessed article. More recently, the journal featured two papers looking at drug repurposing options for $\mathrm{AD}$, uniquely combining empirical and real-world evidence (see here and here). One of those generated much interest online and is currently at the top of the list of Nature Aging papers tracked by Altmetrics, ranking approximately 100th among over 330,000 articles of a similar age.

Another major disease associated with aging is COVID-19. Studies published in the journal have contributed to a better understanding of the disease, including its biology and the strong association of its severity with age. A Review presented a comprehensive overview of the response of the aging immune system to SARS-CoV-2 and a Meeting Report highlighted some of the current gaps in knowledge to be addressed in the future. Other studies have looked at the effect of the disease on our society: for instance, by evaluating the toll on mental health in older populations or by determining some of the factors associated with disease outcomes in long-term care settings. One study successfully tested an informational intervention on older adults to increase the perceived risk of viral transmission, with possible implications for curbing the spread of other infectious diseases such as influenza.

The social sciences were also prominently featured, including in a special issue on ageism in February 2021. An economics paper entitled "The economic value of targeting aging”, involving a collaboration between two economists and a biologist, estimated that slowing down aging to increase life expectancy by 1 year could be worth 38 trillion US dollars. A psychology paper in our October issue examined how age influences prosocial behavior across the globe.

The journal has also been a platform for opinions and commentaries. Some of our Editorials advocated for the strengthening of intergenerational connections, the establishment of vaccine mandates in long-term care, and called for increased participant involvement and appropriate representation of the diversity of aging populations in aging research. A World View by the WHO's Dr Tedros introduced a knowledge-exchange platform to support the UN's Decade of Healthy Ageing initiative. Another raised awareness about promoting healthy aging for Indigenous Australian individuals. A position paper advocated for the development of a branch of medicine focused on longevity. A Comment proposed a set of solutions for creating an anti-ageist healthcare system, and another discussed the need for investment in both facility- and home-based long-term care services.

Over the past year, we have routinely issued press releases for papers that we felt would be of interest to the general public. As a result, several articles were discussed in high-circulation newspapers such as the New York Times, The Guardian or the Daily Mail. We have also helped to disseminate all of the results published in the journal on Twitter, where we have accumulated over 8,000 followers. Several articles were also mentioned in Wikipedia pages, influenced policy documents, and led to blog posts and YouTube videos.

Although the journal published its first issue in January 2021, it first opened for submission in early 2020, more-or-less at the start of the pandemic - which at the time did not feel like an auspicious time to embark on a new ambitious project. Little did we know then that the lockdowns, remote work and inability to travel would actually accompany us throughout the launch year and beyond. Assembling an editorial team in this climate was certainly a challenge, but by mid-2020 there were three of us. We never sat in the same room, and yet we all enjoyed working together, albeit remotely, and we all contributed with dedication to building Nature Aging. It is also important to mention the invaluable support we received from the teams in our production, art and press divisions, as well as our assistant and copy editors. Some of the launch manuscript editors have since parted ways with the journal but in the second half of 2021 a new wave of passionate scientists replaced those who left. Our team will further expand in February to help with the growing number of submissions. As editors, what we perhaps missed the most during the pandemic was the opportunity to meet researchers in person at conferences or during local site visits and talk about research and publishing. We look forward to meeting many of you in the months and years to come, including at some of 
the events listed on our recently curated conference page.

In closing, we would like to express our deepest gratitude to all the researchers who believed in the journal early on and trusted us with some of their best work, and to all the reviewers who expertly evaluated some of the submissions we received and helped those papers to improve and reach their potential, here in the journal or elsewhere. Finally, we wish all our readers a very productive research year, full of exciting new discoveries, and we look forward to working with you to publish many of those.

Published online: 20 January 2022

https://doi.org/10.1038/s43587-022-00169-0 